\title{
Surface operators and magnetic degrees of freedom in Yang-Mills theories
}

\author{
A. Di Giacomo \\ University of Pisa and INFN, Sezione di Pisa, \\ Largo Pontecorvo 3, 56127, Pisa, Italy \\ V.I. Zakharov* \\ INFN, Sezione di Pisa, Largo Pontecorvo 3, 56127, Pisa, Italy; \\ ITEP, B. Cheremushkinskaya 25, Moscow, 117218, Russia
}

November 15, 2018

PACS: 12.38.Aw; 11.15.Ha

\begin{abstract}
Magnetic degrees of freedom are manifested through violations of the Bianchi identities and associated with singular fields. Moreover, these singularities should not induce color non-conservation. We argue that the resolution of the constraint is that the singular fields, or defects are Abelian in nature. Recently proposed surface operators seem to represent a general solution to this constraint and can serve as a prototype of magnetic degrees of freedom. Some basic lattice observations, such as the Abelian dominance of the confining fields, are explained then as consequences of the original non-Abelian invariance. Generically, the properties of the two-dimensional defects associated with the surface operators are close to the predictions of the dual models for the magnetic D2 branes. 1
\end{abstract}

*Electronic address: vzakharov@itep.ru

${ }^{1}$ This paper is prepared for the volume dedicated to the 80th birthday of Lev Borisovich Okun. The authors are acknowledging deep influence which Lev's Okun style and works have had on them. One of the main themes of papers of L.B. Okun is that it is only Nature, or experiment which can decide whether a phenomenon is exotic or not, reality or an artefact. We would include nowadays lattice data, or better to say lattice experimentation with field theory into the means to discover new phenomena. Magnetic monopoles were introduced first, as a pure theoretic construct more than 70 years ago by P.M.A Dirac [1]. Reality of descendants of the Dirac monopoles, magnetic degrees of freedom in YangMills theories was established, we believe, via lattice experiment. In these notes we are trying to make a step towards theoretical interpretation of these magnetic degrees of freedom invoking for this purpose 


\section{Introduction}

It is commonly accepted that confinement is due to condensation of magnetic degrees of freedom. Such a mechanism is established, both theoretically and via lattice measurements in the Abelian cases of a pure gauge field in the compact version of the theory and $Z_{2}$ gauge theory, for an early review see [3]. In this case the magnetic degrees of freedom are in fact the Dirac monopoles.

Moreover on the lattice one observes monopole trajectories and has to use the polymer approach to field theory, see, e.g. 4, to describe the monopole properties in the language of the quantum geometry. In the geometrical language the confining field configurations are identified as infinite clusters of $1 \mathrm{~d}$ defects, or trajectories. Another Abelian example is the $Z_{2}$ gauge theory. In this case the confining configuration is a percolating cluster of surfaces, or vortices.

In the non-Abelian case 2 there is no consensus yet on the particular choice of the magnetic degrees of freedom. The general idea is to reduce the non-Abelian degrees of freedom to the pattern of Abelian theories where the mechanism of confinement is well understood. For this purpose one introduces the so called projected fields:

$$
A_{\mu}^{a}(x) \rightarrow A_{\mu}^{3}(x), \text { or } A_{\mu}^{a}(x) \rightarrow\left(Z_{2}\right)_{\mu}(x)
$$

where $A_{\mu}^{3}(x)$ is an Abelian gauge field, and $\left(Z_{2}\right)_{\mu}(x)$ is a $Z_{2}$ gauge field $\left(\left(Z_{2}\right)_{\mu}(x)=\right.$ \pm 1 for links). The $\mathrm{U}(1)$ projections emphasize the role of the monopole trajectories or $1 \mathrm{~d}$ defects while $Z_{2}$ projections allow to find center vortices, or $2 \mathrm{~d}$ defects 3 .

The projected fields (1) are related to the original Yang-Mills fields in a nonlocal way and theoretical interpretation of the lattice data is obscured by their use. It turns impossible to reconstruct configurations of the original non-Abelian fields $A_{\mu}^{a}(x)$ which correspond to the monopoles or vortices. However, it seems indisputable that effectively Abelian degrees of freedom are responsible for the confinement.

In this note we will reverse the problem and instead of trying to directly interpret the lattice data consider classification of non-Abelian singular fields in the continuum which could be responsible for violations of the Bianchi identities while not inducing violations of the color conservation. We argue that a natural candidate for such defects are singular fields living on two-dimensional surfaces. The central point is that such fields are Abelian in nature and, therefore, violations of the Bianchi identities and of the equations of motion are disentangled.

the notion of surface operators [2] which proved useful in such mathematical discipline as number theory. We are amused by the idea that a kind of Abelian dominance exists both in Yang-Mills theory and in the number theory and the same mathematical means can be adapted to describe the two phenomena. We hope that the simplicity of the basic means used will match Lev's Okun quest for clarity of the basic concepts of physics.

${ }^{2}$ For simplicity we consider pure SU(2) gauge theory, without matter.

${ }^{3}$ The literature on the confinement is vast. For the purpose of orientation and further references we can mention reviews in Ref. [5] and in Ref. [6] exposing in detail the $U(1)$ and $Z_{2}$ projections, respectively. 
As far as the mathematical tools are concerned, we utilize the so called surface operators 2 which describe four-dimensional (4d) non-Abelian fields living on $2 \mathrm{~d}$ surfaces. It is interesting that from the phenomenological point of view the use of the surface operators suggests a unification of the two alternative Abelian pictures mentioned above. Namely, the defects are $2 \mathrm{~d}$, as emphasized by the center projections, while the fields living on them are Abelian, as commonly emphasized by the $U(1)$ projections.

\section{Abelian case}

To set up the framework, let us review first the compact $U(1)$ theory [7]. The Lagrangian is the same as for a free electromagnetic field:

$$
L_{U(1)}=\frac{1}{4 e^{2}}\left(F_{\mu \nu}\right)^{2},
$$

supplemented, however, by the condition that the Dirac string carries no action. The condition is automatically satisfied in the lattice version of the theory.

Admitting singular fields, or monopoles into the theory violates Bianchi identities and the modified Maxwell equations now read:

$$
\partial_{\mu} F_{\mu \nu}=0, \partial_{\mu} \tilde{F}_{\mu \nu} \equiv j_{\nu}^{m o n}, \partial_{\nu} j_{\nu}^{m o n}=0 .
$$

where $j_{\nu}^{\text {mon }}$ is the monopole current.

The non-vanishing, conserved current $j_{\nu}^{\text {mon }}$ can be traded for a magnetically charged scalar field $\phi_{M}$. This is a generic field theoretic phenomenon (in the Euclidean space-time). The derivation can be found in quantum-geometry courses, see, e.g., 4] while specific applications to lattice monopoles are discussed, in particular, in Refs [8]. Here we will remind, for a later use, a few basic steps in relating the monopole current to a magnetically charged field.

Observing $j_{\mu}^{\text {mon }}$, say, in lattice simulations is equivalent to observing particle trajectories. Therefore, it is reasonable to start from the so called polymer formulation of a free scalar field theory with the action

$$
S_{c l}=M_{\text {bare }} \cdot L,
$$

where $M_{\text {bare }}$ is the (bare) mass and $L$ is the length of trajectory.

The mapping of the polymer representation (44) into the standard field theoretic representation is achieved through evaluating the path integral for the particle propagator:

$$
D(x, y, M)=\Sigma_{\text {paths }} \exp \left(-S_{c l}(x, y, M)\right) .
$$

The sum (5) can be evaluated exactly and one establishes [4] a relation between the physical mass $m_{\phi}^{2}$ and the polymer-representation mass $M$ :

$$
m_{\phi}^{2} \approx \frac{\text { const }}{a}\left(M_{\text {bare }}(a)-\frac{\ln 7}{a}\right),
$$


where $\ln 7$ is a geometric factor specific for a cubic lattice in $d=4$ and we introduced explicitly dependence of the bare mass (4) on the lattice spacing $a$ which is an ultraviolet cut off.

To relate the polymer approach to the physics of the lattice monopoles one identifies the bare mass $M_{\text {bare }}$ in (4) with the radiative mass of the monopole:

$$
M_{\text {bare }} \rightarrow M_{m o n}=\frac{\text { const }}{a e^{2}},
$$

where $e^{2}$ is the electric charge squared. The Higgs, or confining phase corresponds to $m_{\phi}^{2}<0$. Once $m_{\phi}^{2}=0$ there emerges an infinite, or percolating cluster of the monopole trajectories. The relation between the monopole density $\rho_{m o n}$ and the field-theoretic vacuum expectation value reads as:

$$
\left\langle\left. 0|| \phi\right|^{2} \mid 0\right\rangle=\text { const } \cdot a \rho_{\text {mon }}, \quad(\langle 0|\phi| 0\rangle)^{2}=\text { const } \cdot a \rho_{\text {mon }}^{\text {perc }},
$$

where $\rho_{\text {mon }}$ is the total monopole density and $\rho_{m o n}^{\text {perc }}$ is the density of the percolating monopoles.

\section{Non-Abelian singular fields}

The Abelian construction just described does not generalize to the non-Abelian case. Indeed, if

$$
\left(D_{\mu} \tilde{G}_{\mu \nu}\right)^{a}=j_{\nu}^{a},
$$

where $j_{\nu}^{a}$ is the monopole current, then the colored current $j_{\nu}^{a}$ would modify also the equations of motion, not only the Bianchi identities since any colored current is a source of gluons. Moreover, if we trade the current $j_{\nu}^{a} \neq 0$ for a scalar field then this field is colored, $\phi^{a}$ and its vacuum expectation value would violate color conservation.

Now, we are coming to a crucial point. We do not take these difficulties as a proof that singular fields have no role to play in the non-Abelian case. Instead, we merely conclude that trajectories, or $1 \mathrm{~d}$ defects are not adequate to the non-Abelian case and will be looking for defects of other dimensions. Note that the fact that monopoles are intrinsically $\mathrm{U}(1)$ (not $S U(2)$ ) objects has been emphasized since long, see, in particular, [9].

Turn now to two-dimensional defects, or surfaces. Classification of singular field living on a surface, or $2 \mathrm{~d}$ defects is actually contained in [2 4. The central point is that non-Abelian fields living on a surface can in fact be rotated to an Abelian direction and as a result violations of the Bianchi identities $\left(D_{\mu} \tilde{G}_{\mu \nu} \neq 0\right)$ can be consistent with the validity of the equations of motion $\left(D_{\mu} G_{\mu \nu}=0\right)$.

\footnotetext{
${ }^{4}$ The observation on Abelian nature of the non-Abelian fields living on a surface, crucial for our considerations, was intensely exploited also earlier [10. However, the surface operators also allow to ascribe to the surfaces density of topological charge, see below, and phenomenological consequences from this observation have not been considered, to our knowledge.
} 
Introduce first the action associated with the surfaces in the form:

$$
S_{\text {surface }}=\text { const } \int d \sigma_{\mu \nu} G_{\mu \nu}^{a}
$$

This action is not gauge invariant. However, it can be redefined in such a way as to respect the non-Abelian invariance. The reason is that the surface interaction at each point $x$ involves only a single component of the field strength tensor $G_{\mu \nu}^{a}$. Therefore, one can use gauge invariance to rotate this particular component to the Cartan subgroup:

$$
d \sigma_{\mu \nu} G_{\mu \nu}^{a}(x) \rightarrow d \sigma_{\mu \nu} G_{\mu \nu}^{3}(x),
$$

where for simplicity we considered the gauge group $S U(2)$.

Note that the projection (9) is determined up to a sign. One can fix the sign by imposing the condition

$$
\left(d \sigma_{\mu \nu} G_{\mu \nu}^{3}\right)(x) \cdot\left(d \sigma_{\mu \nu} G_{\mu \nu}^{3}\right)(y)>0 .
$$

As is argued in 2] the surface can be endowed also with a dual field $\tilde{G}_{\mu \nu}^{a}$ which can also be rotated to the Cartan subgroup:

$$
d \sigma_{\mu \nu} \tilde{G}_{\mu \nu}^{a}(x) \rightarrow d \sigma_{\mu \nu} \tilde{G}_{\mu \nu}^{3}(x),
$$

where the point $x$ belongs to the surface. Note that only one of the two rotations (9), (11) is uncertain in sign.

From the point of view of the lattice formulation, the possibility of two independent (apart from a sign) rotations assumes a particular regularization. Indeed, the dual field is defined on the dual-lattice plaquettes and in this sense can be rotated independently. However, such a procedure would assume simultaneous use of both direct and dual lattices which is not necessarily legitimate. We will not go into details of this issue here and just follow Ref. [2] in postulating, in the continuumtheory language, existence of closed surfaces, with surface element $d \sigma_{\mu \nu}$, and with the fields $G_{\mu \nu}^{3}, \tilde{G}_{\mu \nu}^{3}$ associated with the surfaces. One ascribes then to the surface the following action

$$
S_{\text {surface }}=\alpha \int d \sigma_{\mu \nu} G_{\mu \nu}^{3}+\beta \int d \sigma_{\mu \nu} \tilde{G}_{\mu \nu}^{3}
$$

where $\alpha, \beta$ are constants.

Note that in terms of invariants the two-dimensional defects considered have both non-vanishing action and topological-charge densities,

$$
G^{2}(x)>0, \quad G \tilde{G}(x) \neq 0,
$$

as far as the point $x$ belongs to the surface. The fields $G_{\mu \nu}^{3}, \tilde{G}_{\mu \nu}^{3}$ can be directly defined in terms of the invariants as

$$
G_{\mu \nu}^{3}(x) \equiv+\sqrt{G^{2}(x)} \frac{d \sigma_{\mu \nu}}{\left|d \sigma_{\mu \nu}\right|}, \quad \tilde{G}_{\mu \nu}^{3}(x) \equiv \frac{G \tilde{G}(x)}{\sqrt{G^{2}(x)}} \frac{d \sigma_{\mu \nu}}{\left|d \sigma_{\mu \nu}\right|},
$$

where the point $x$ belongs to the surface. 


\section{Various facets of defects}

\subsection{Wilson lines}

Two-dimensional surfaces introduced above can be considered on classical or quantum level, as external objects or as dynamical degrees of freedom. Indeed, turn first to the example of the Wilson line. Classically (and in Abelian case)

$$
\int_{C} A_{\mu} d x_{\mu}=\int_{\text {surface }} d \sigma_{\mu \nu} H_{\mu \nu}
$$

where the surface is spanned on the contour $C$. In the classical case the contour integral merely 'measures' the external magnetic flux. If the potential $A_{\mu}$ is given locally by a pure gauge, the contour integral is quantized.

If we introduce the Wilson line as an external object and consider it quantum mechanically, then there emerge divergences due to the self energy:

$$
\left\langle\operatorname{Tr} P \exp \left\{-\int_{C} \hat{A}_{\mu} d x_{\mu}\right\}\right\rangle \sim \exp \left(- \text { const } g^{2} L / a\right),
$$

where $L$ is the perimeter of the Wilson line $C, a$ is the lattice spacing, $g^{2}$ is a coupling and we keep only the most divergent piece.

Finally, one can consider the loops as dynamical objects, generated within the theory itself. In particular, it is quite common nowadays to consider a condensate of Polyakov's lines [11] which are defined at finite temperature:

$$
\operatorname{Tr} L \equiv \operatorname{Tr} P \exp \left\{-\int_{0}^{1 / T} d x_{0} \hat{A}_{0}(\mathbf{x}) d x_{0}\right\}
$$

and are the Wilson lines stretched in the time direction.

Phenomenologically, it is appealing to assume that there exists effective potential for the vacuum expectation value of the Polyakov's lines:

$$
V(<\operatorname{tr} L>)=-c_{2}<\operatorname{tr} L>^{2}+c_{4}<\operatorname{Tr} L>^{4},
$$

where in the deconfining phase, or at $T>T_{c}$ the constants $c_{2,4}$ are positive, see, in particular [12].

Let us emphasize that for the vacuum expectation value $<\operatorname{Tr} L>$ to be nonvanishing,

$$
<\operatorname{Tr} L>\neq 0
$$

the ultraviolet divergence exhibited by (15) is to be canceled by the entropy. We discussed above such a cancelation on the example of theory of a free particle within the polymer approach. 


\subsection{Surface integrals classically}

Surface integrals similar to (12) appear in the GNO classification [15] of the nonAbelian monopoles 5. One assumes that there exist classical solutions such that at large distances $r$ from the position of the monopole the (space-space) components of the gauge field tensor take the form

$$
G_{i j}=\frac{\epsilon_{i j k} r_{k}}{r^{3}} G(r)
$$

where $G(r)$ are magnetic charges. The solution can possess an electric charge as well and then the dual field $\tilde{G}_{i j}$ is also non-vanishing. The observation [15] is that in gauge theories, though electric charge takes values in the weight lattice of the gauge group, the (quantized) magnetic charge $G$ takes values in the weight lattice of a dual group.

In case of the gauge group $S U(N)$ the dual group is $S U(N) / Z_{N}$ where $Z_{N}$ is the center group. In the simplest case of $\mathrm{SU}(2)$ group which we concentrate on the magnetic charges $Q_{M}= \pm 1$ are to be identified since the corresponding magnetic fields (17) can actually be gauged transformed into each other.

\subsection{Surface operators as external objects}

As is mentioned above, Ref. [2] introduces the surface operators as external objects on the quantum level. One can measure vacuum expectations value of the surface operators by substituting the the vacuum fields (14) defined for each configuration on the lattice and then averaging over the configurations.

Clearly, the vacuum expectation value is suppressed by the ultraviolet divergent self energy proportional to the area of the closed surface:

$$
\langle S\rangle \sim \exp \left(-\alpha \cdot(\text { const })(\text { Area }) / a^{2}\right)
$$

where $\alpha$ enters definition of the action (12) and (const) is related to the average value of the plaquette action. In other words, $G_{\mu \nu}^{3}(x)$ living on the surface appears singular in the limit of the vanishing lattice spacing $a \rightarrow 0$. The divergence corresponds to self energy of colored dipoles living on the surface and oriented in the third direction of the color space. It is an analog of the divergence (15) in case of the Wilson lines.

One can measure also density of the topological charge $G \tilde{G}(x)$ for the points $x$ belonging to the surface, $x \epsilon \Sigma$. The results of the measurements would depend on the properties of the Yang-Mills vacuum and cannot immediately be predicted.

As in case of the Wilson line one can consider quantities not sensitive to the ultraviolet divergence (18). For this purpose one can consider, for example, closed surfaces unifying two planes separated by distances $r$ and measure potential energy as function of $r$. Studying expectation values of the surface operators could probe the confinement, similar to the case of the Wilson lines [2]. Indeed, the vacuum

\footnotetext{
${ }^{5}$ The surfaces are closed in this case. Hereafter we will consider closed surfaces in all the cases.
} 
expectation value of the surface operator (apart from the just mentioned ultraviolet divergent piece) might exhibit either volume or area laws:

$$
\langle S\rangle \sim \exp (-(\text { const })(\text { Volume })), \text { or }\langle S\rangle \sim \exp (-(\text { const })(\text { Area })) .
$$

Note that by measuring the vacuum expectation values of the surface operators one studies the interaction of particles having color magnetic and dipole moments, but no color charge. Therefore, it is our guess that there is no volume law even in the confining phase.

\subsection{Surfaces as dynamical defects}

In this paper we are going to make the next step and assume that surfaces become dynamical and condense in the vacuum in the confining phase so that there is a net of surfaces endowed with fields living on them. The picture is similar to condensation of the Polyakov's lines in the deconfinement phase.

It is a strong assumption concerning the dynamics of non-Abelian theories which in no way can be derived from first principles. Our assumption is motivated by the lattice data that do demonstrate existence of $2 \mathrm{~d}$ surfaces percolating through the vacuum [6, 13]. For a more theoretically oriented reader let us mention that holographic models predict both percolation of the Polyakov's lines in the deconfining phase and percolation of magnetic strings in the confining phase [14.

In more detail, we will rely on the Sakai-Sugimoto holographic model [16] which is most successful in describing low-energy QCD. The metrics of this model is formulated in terms of the ordinary $4 \mathrm{~d}$ space $\left(t, x^{i}, i=1,2,3\right)$, extra fifth dimension $u$ with horizon at $u=u_{\Lambda}$, one more extra compact dimension $x^{4}$ and compact sphere $\Omega_{4}$ :

$$
\begin{array}{r}
d s^{2}=\left(\frac{u}{R_{0}}\right)^{3 / 2}\left(-d t^{2}+\delta_{i j} d x^{i} d x^{j}+f(u) d x_{4}^{2}\right)+\left(\frac{u}{R_{0}}\right)^{-3 / 2}\left(\frac{d u^{2}}{f(u)}+u^{2} d \Omega_{4}^{2}\right) \\
f(u)=1-\left(\frac{u_{\Lambda}}{u}\right)^{3} .
\end{array}
$$

It is also crucial that the defects wrapped on the compact dimension $x^{4}$ have nontrivial $\theta$-angle dependence. Note that the metric in dimensions $\left(x^{4}, u\right)$ has a cigar form so that the radius of the $x^{4}$ compact dimension tends to zero at the horizon $u=u_{\Lambda}$. Which means that the defects wrapped around this dimension have tension vanishing at the horizon. In particular, the magnetic strings are defined as D2 branes wrapped around the $x^{4}$ dimension. They look as strings, or $2 \mathrm{~d}$ surfaces in the ordinary $4 \mathrm{~d}$ space and, in case of large enough area, have vanishing (classically) tension. Because the D2 branes are wrapped on the compact $x^{4}$ dimension one expects that they carry density of the topological charge.

The metrics (20) refers to the case of zero temperature $T=0$ which we are mostly interested in. At finite $\mathrm{T}$ it is convenient to use Euclidean space, with a compact time direction $\tau$. Thus, at $T \neq 0$ there are two compact coordinates, $x^{4}$ 
and $\tau$. The deconfining phase transition is then understood as change of geometries in two-dimensional $\left(x^{4}, u\right)$ and $(\tau, u)$ subspaces. Namely, at $T>T_{c}$ the cigarshape geometry sets in the $\tau, u$ coordinates while in the $\left(x^{4}, u\right)$ coordinates the geometry becomes cylinder-like. As a result, defects wrapped in (Euclidean) time direction become tensionless and could condense. As is argued in 14 this is a general phenomenon within the dual model: below $T_{c}$ defects condensed in the vacuum have nontrivial $\theta$-angle dependencies while at $T>T_{c}$ it is the defects wrapped on the compact time direction which are tensionless. The Polyakov line is wrapped around the time direction and becomes tensionless at $T>T_{c}$ according to the dual model. This fits well the phenomenological models which postulate condensate of the Polyakov's lines. The magnetic D2 branes are wrapped around the compact $x^{4}$ direction and are tensionless at small temperatures. They are expected to condense at small $\mathrm{T}$.

\section{$5 \quad$ Surface operators and magnetic branes}

The hint which we get from the dual models is that in the Yang-Mills vacuum there could exist magnetic strings, tensionless. We conclude that their tensionlessness is to be manifested as percolation of $2 \mathrm{~d}$ surfaces, or existence of an infinite cluster of such vortices. As usual in quantum geometry, the surfaces are to be endowed with action. Moreover, the vanishing string tension implies action-entropy balance. Another important prediction of the dual models is that the surfaces are carrying topological charge density (since the D2 branes are wrapped around the compact $x^{4}$ coordinate).

A crucial observation is that the surface operators are an adequate mathematical tool to consider the magnetic strings because they also describe $2 \mathrm{~d}$ surfaces endowed both with action and topological charge. In this section we will consider further phenomenological consequences of such identification.

\subsection{Non-Abelian 'monopoles'}

Assumption on the dynamical nature of the $2 \mathrm{~d}$ defects brings about a conclusion that there should exist also 1d defects living on surfaces. Indeed, consider first two external surfaces with

$$
\left(G^{2}\right)_{1}=\left(G^{2}\right)_{2},(\tilde{G} G)_{1}=-(\tilde{G} G)_{2} .
$$

As far as the surfaces are treated as external objects they are different although degenerate in energy. However, once we allow for dynamical surfaces to exist the two degenerate states mix up and the actual ground state would consist of their mixture. Lines on which the $\tilde{G} G$ changes its sign are one-dimensional defects. Which we will call non-Abelian monopoles (since they are associated with lines, or trajectories). 
There is an important difference in the structure of gauge fields associated with the Abelian and non-Abelian monopoles. Consider the Abelian case first and introduce a time slice of the $4 \mathrm{~d}$ space. Then at some point a singular magnetic field may not have a particular direction:

$$
\mathbf{H}_{\text {Abelian }} \sim \frac{\mathbf{r}}{r^{3}},
$$

where $\mathbf{r}=0$ is the position of the singularity. Such a singularity corresponds to the Dirac monopole.

In the non-Abelian case consider a time slice of the surface which is a line, with coordinate $\tau$. At some point $\tau_{0}$ the magnetic field living on the line may change direction:

$$
\mathbf{H}_{\text {non-Abelian }}=|\mathbf{H}| \frac{\left(\tau-\tau_{0}\right)}{\left|\tau-\tau_{0}\right|} .
$$

It is crucial that the magnetic field in the non-Abelian case is not spherically symmetric but is line-like. Such field configuration is allowed by the non-Abelian invariance as a defect while the spherically symmetric field (21), familiar from the Abelian case, is not allowed. If we now include development in time both the Abelian and non-Abelian monopoles become lines, or trajectories. However, in the Abelian case the trajectories live on $4 \mathrm{~d}$ space while in the non-Ableian case they live on $2 \mathrm{~d}$ surfaces.

Let us emphasize that the very existence of the $1 \mathrm{~d}$ defects depends crucially on the assumption that the $2 \mathrm{~d}$ defects (surfaces) carry both fields $G_{\mu \nu}^{3}$ and $\tilde{G}_{\mu \nu}^{3}$. If there were only magnetic fields living on the surface the change of the direction of the magnetic field, see Eq. (22) would be obscured by the non-Abelian gauge invariance which allows to change the sign of the magnetic field.

\section{$5.2 \quad$ Open strings}

So far we were discussing closed surfaces, or strings. One might ask whether it is possible to have an open string as a defect. Thus, we wish to introduce a $1 \mathrm{~d}$ boundary. For the whole construction to be non-Abelian invariant, this boundary, or line is to have also non-Abelian invariant meaning. Physicswise, the magnetic field which exists along the strings 'flows' to its end in case of an open string. Thus, it is natural to have a monopole at the end points. Moreover, this 'monopole' is to be defined in an $S U(2)$ invariant way. There is only a single monopole of this type, that is the $Z_{2}$ monopole [18, or the 't Hooft line [19] (in case of $S U(N)$ group there are $(N-1)$ species of $Z_{N}$ monopoles).

The $Z_{2}$ monopole can be viewed also as an ordinary Abelian monopole with magnetic flux $2 \pi / g$ :

$$
\int_{Z_{2} \text { monopole }} d \sigma_{\alpha \beta} G_{\alpha \beta}=\frac{2 \pi}{g}
$$

where $g$ is the gauge coupling. Note that the corresponding Dirac string is having quadratically divergent action and in this sense is visible (for further discussion 
see [20]). Finally, we note that the magnetic field living on the $2 \mathrm{~d}$ surface is not quantized and the magnetic flux flowing to the end points of the string is generically not equal to the flux (23).

\section{Lattice data}

As we have already mentioned, the lattice data demonstrate existence of $2 \mathrm{~d}$ surfaces with properties close to the predicted above. Mini-reviews of the data can be found, e.g., in Ref. [13]. For the sake of completeness, we reiterate main points here.

Definition of the surfaces on the lattice are algorithmic and is difficult to translate into the continuum-theory language. This is the main difficulty in interpretation of the data. As a partial compensation for this difficulty the lattice itself allows for specific and powerful tests of the hypothesis that some particular lattice defects have meaning in the continuum limit. First of all, one can check scaling laws which are obeyed by physical objects, for reviews see [6, 5 .

Let us give an example. Imagine that there is a technical definition of closed $2 \mathrm{~d}$ surfaces in Yang-Mills vacuum. One can measure global characteristics of it. In particular, one measures the total area of the surface. Imagine, furthermore, that one finds:

$$
(\text { Area })_{\text {tot }} \approx \Lambda_{Q C D}^{2} V_{\text {tot }},
$$

where $V_{t o t}$ is the total volume of the lattice. The proportionality to $V_{t o t}$ is triviality for large enough volumes. However, the proportionality to the physical scale, $\Lambda_{Q C D}^{2}$ is highly nontrivial.

On the lattice, one changes the lattice spacing $a$ and the bare coupling $g^{2}(a)$, in accordance with the renormgroup. Absence of explicit dependence of the area (24) on the lattice spacing implies strong 'conspiracy' of the data. Indeed the statement (24) can be rewritten as observation that probability of a given plaquette to belong to the surface is proportional to

$$
\theta_{\text {plaq }} \approx \operatorname{const}\left(a \cdot \Lambda_{Q C D}\right)^{2}
$$

and changing the lattice spacing by, say, factor of 2 changes the probability (25) by a factor of 4 which is a well defined prediction and a large effect numerically.

Once relation (24) is found empirically, in terms of a certain $Z_{2}$ projection of the original non-Abelian fields it becomes a strong evidence that using this projection allows to detect a gauge invariant physical object. Indeed, the property (24) is perfectly gauge invariant. Moreover, it implies that the tension of the strings is in physical units:

$$
T_{\text {string }} \sim \Lambda_{Q C D}^{2},
$$

since it is the tension which controls the area. Of course it is not a complete proof yet of the physical nature of the surfaces. Moreover, one could reverse the procedure and just try to define such a projection that Eq. (24) holds with good accuracy. 
Thus, we trade the property (24) for the definition of the surface. The central problem then, are there further gauge invariant properties possessed by the surface. In case of the center vortices the answer is in positive (for review and details see [6, 13]).

In particular, there is an extra non-Abelain action associated with the surfaces 20]:

$$
\delta S_{\text {surface }} \approx(\text { const }) \frac{(\text { Area })}{a^{2}},
$$

where the use of the symbol 'approximate' always means that the evidence is numerical, i.e., true within some error bars. We do not discuss the error bars here and only sketch the overall picture.

The physical meaning of (27) is that the construction is consistent at short distances. Indeed, in the language of the quantum geometry having (26) is consistent only if there is action-entropy balance:

$$
(\text { Tension })(\text { Area })=(\text { Action })_{\text {surface }}-(\text { Entropy })_{\text {surface }}
$$

where both the action and entropy associated with the surface are ultraviolet divergent while their difference is finite.

Another confirmation of this amusing picture is provided by measurement of the extra action associated with plaquettes next to those belonging to our surfaces. It vanishes identically:

$$
\delta S_{\text {next to surface }}=0 \text {. }
$$

In physical terms, it is a confirmation that the action is indeed associated with the field living on the surface as we have been conjecturing.

A crucial property of the surfaces we are discussing is that they are expected to possess not only action but density of the topological charge as well. Amusingly enough, this prediction has already been tested and, within error bars, confirmed. Namely, one measures on the lattice for each particular configuration space distribution of wave functions of topological modes of quarks. It turns out that the intensity of the topological charge (as measured by intensity of the topological fermionic modes) is correlated positively with the position of the surfaces and the correlation grows as an inverse power of the lattice spacing [21]:

$$
\left\{(\text { Intensity (topological modes), Intensity (surfaces) }\} \sim a^{-\gamma},\right.
$$

where the critical exponent $\gamma \approx 26$.

Apart from the surfaces, or $2 \mathrm{~d}$ defects one observes on the lattice $1 \mathrm{~d}$ defects, or monopole trajectories as well. The monopoles observed on the lattice 7 do seem to have properties close to those predicted theoretically. Namely, they lie on the surfaces just discussed and their non-Abelian field is rather line-like, aligned with the surfaces.

\footnotetext{
${ }^{6}$ The precise definition of the correlator (30) is rather technical in nature. It can be found in the original papers 21 .

${ }^{7}$ So called monopoles of the Maximal Abelian Projection.
} 


\subsection{Localization of classical solutions}

Thus, the center vortices on the lattice do have properties similar to those inherent to the surfaces discussed in the preceding sections and based on the surface operators. However, the reader might feel uncomfortable to discuss confinement in terms of singular fields (18). Indeed, in the continuum theory one usually discusses nonperturbative physics in Yang-Mills theory in terms of soft fields. On the other hand, the lattice data reveals that hard fields are associated with the confinement and this cannot be disregarded. Thus, we have a paradox.

It is worth emphasizing therefore that it would be wrong to consider the infrared and ultraviolet sensitive descriptions as mutually excluding each other. It is much more adequate to think in terms of a kind of duality, that the nonperturbative physics can be described either in infrared or ultraviolet terms. Moreover, one description probably smoothly goes into the other one as a functions of resolution of measurements, or of the value of the lattice spacing [22]. The term 'resolution' is used here because generating each filed configuration on the lattice, $\left\{A_{\mu}^{a}(x)\right\}$ can be considered as a measurement of the gauge fields on the whole lattice with resolution of the lattice spacing $a$.

Unfortunately, there is no detailed understanding of the role of the resolution. Probably, one deals with quite a general phenomenon which can be called stabilization of classical solutions with high-frequency oscillating fields. At this time the guess can be substantiated, however, only by some analogies and intuitive considerations. It would be extremely interesting to clarify further these issues 8 .

Let us start with a far fetched analogy from classical mechanics. Consider a pendulum with frequency $\Omega_{\text {slow }}$. Apply now a fast oscillating force to the fixed point of the pendulum, with amplitude $a_{\text {fast }}$ and frequency $\omega_{\text {fast }}$. Then the effective potential which determines the resulting motion of the pendulum becomes:

$$
U_{\text {eff }}=m g l(1-\cos \phi)+a_{\text {fast }}^{2} \omega_{\text {fast }}^{2} \sin ^{2} \phi
$$

where $\phi$ is the angle of deviation of the pendulum from the vertical line.

If the ratio $\omega_{\text {fast }} / \Omega_{\text {slow }}$ is large the effective potential (31) is much steeper than the original one. The resulting amplitude of oscillation of the pendulum around $\phi=0$ is much smaller than without the oscillating force. Moreover, the point $\phi=\pi$ becomes a minimum of the effective potential as well 9 .

In the Yang-Mills case, the analogy to slow motion is provided by a classical solution, say instanton, of size $\rho_{\text {inst }} \sim \Lambda_{Q C D}^{-1}$. Imagine that we would like to next

\footnotetext{
${ }^{8}$ Analytically, interplay between the infrared and ultraviolet sensitive descriptions was discovered first in $2 \mathrm{~d} \mathrm{CP}(\mathrm{N})$ models 24 . Namely, it was proven that upon summation over all the instanton solutions (including multi-instantons) the instanton ensemble is described by the Coulomb-gas model. The constituents are point-like and possess topological charge equal to $1 / N_{c}$ fraction of that of the instanton.

${ }^{9}$ This is a famous example due to S. Kapitza, see L.D. Landau and E.M. Lifshitz, Course of Theoretical Physics, vol. 1.
} 
include effect of zero-point fluctuations which are fast oscillating fields. The zeropoint fluctuations are dominated by the ultraviolet scale:

$$
\left[a_{\mu}^{a}\right]_{\text {quant }} \sim a_{\text {latt }}^{-1}
$$

where $a_{\mu}^{a}$ is the quantum gauge field and $a_{\text {latt }}$ is the lattice spacing.

The classical instanton solution $\left(A_{\mu}^{a}\right)_{\text {class }}$ is no longer a solution. Indeed $\left(A_{\mu}^{a}\right)_{\text {class }} \sim$ $\rho_{\text {inst }} / g$ and is much smaller, by the factor of order $\left(a_{\text {latt }} / \rho_{\text {inst }}\right)$ than the quantum field $a_{\mu}^{a}$. In analogy with the mechanical example mentioned above one should rather first average over fast quantum oscillations and derive an effective potential.

Of course, there are no means to solve this problem analytically. However, it seems rather obvious that the 'instanton' shrinks, the same as the motion of the pendulum. Indeed, instantons are in fact generic non-perturbative fluctuations and the probability to find such a fluctuation is of order

$$
\theta_{\text {non-pert }} \sim \exp \left(-(\text { const }) / \alpha_{s}\left(a_{\text {latt }}^{2}\right)\right),
$$

where $\alpha_{s}\left(a_{\text {latt }}^{2}\right)$ is the running coupling. The crucial point is that $\alpha_{s}$ is normalized on the scale on which measurements are performed, i.e. on the scale $a_{\text {latt }}$.

Estimate (32) implies that the instanton volume shrinks to zero as a power of the lattice spacing $a_{\text {latt }}$. Moreover, relying bluntly on the analogy with the pendulum, we come to a bizarre conclusion that

$$
V_{\text {instanton }} \sim(\text { const }) \cdot a_{\text {lattice }}^{4} .
$$

Strange to say, but this guess might well be true, according to the lattice data 10 .

Let us specify the notion of the 'volume occupied by an instanton'. The definition is provided actually by considering zero- or near-zero fermionic modes. In the classical instanton case the topological modes are located in the same region of the $4 \mathrm{~d}$ space as the instanton itself:

$$
\left|q_{\lambda=0}(x)\right|^{2} \sim \frac{\rho^{4}}{\left(\rho^{2}+x^{2}\right)^{3}}
$$

where $q_{\lambda=0}$ is a zero mode of the Dirac equation for a quark in external gluon field:

$$
\hat{D_{\mu}} \gamma_{\mu} q_{\lambda=0}=0
$$

and to derive (34) one substitutes the instanton field for the external gluon field.

On the lattice, one can solve the Dirac equation numerically substituting into the covariant derivative $D_{\mu}$ the gluon fields generated on the lattice for a particular configuration $\left\{A_{\mu}^{a}(x)\right\}$. Then the volume occupied by the topological fermionic

10 The phenomenon of the shrinking of classical solutions was discovered by the ITEP lattice group [20. However, the classical solutions relevant to this paper belong to a dual formulation of the Yang-Mills theories and the status of these solutions is rather vague. The idea to check this phenomenon on the case of 'instantons', or chiral defects belongs to the authors of the first paper in Ref. [23]. 
modes can be understood as the volume occupied by 'instanton modified by highfrequency perturbative fields'. Lattice data rather support estimate (33).

Let us emphasize that the shrinking volume of the instanton implies also singular fields inside the small region occupied by the modified instanton. Indeed, the average value of the topological charge squared, or topological susceptibility,

$$
\int d^{4} x<0|G \tilde{G}(x), G \tilde{G}| 0>\sim \Lambda_{Q C D}^{4},
$$

represents a matrix element and its value cannot depend on details of the measurements, in particular on the value of $a_{\text {latt }}$. On the other hand, the value of $Q_{t o p}^{2}$ for each configuration is equal to the number of exact zero modes of the quarks. Thus, the total topological charge of configurations cannot depend on the shrinking of each particular lump of the topological charge. Hence, the topological fields become singular in the limit of the vanishing volume of the lumps.

Assuming all this to be correct, what are implications for interpretation of $2 \mathrm{~d}$ surfaces? To answer the question note that in terms of the dual model instantons are D0 branes. These branes have one extended coordinate which is wrapped around the $x^{4}$ circle. In the geometrical language of the dual models (20) this wrapping is responsible for the non-trivial topological charge of instantons. To summarize: the instantons are points in ordinary $4 \mathrm{~d}$ space, within the defects classification of the dual models.

On the other hand, in the ordinary field theoretic language the instantons are rather extended objects of the size of order $\rho \sim \Lambda_{Q C D}^{-1}$. Remarkably enough, the shrinking caused by $a_{\text {latt }} \rightarrow 0$ just brings an instanton to a point:

$$
\left(G^{2} \sim \frac{\rho^{4}}{\left(\rho^{2}+x^{2}\right)^{4}}\right) \rightarrow\left(G^{2} \sim \delta^{4}(x)\right), a_{\text {latt }} \rightarrow 0 .
$$

Thus, one can speculate that in the limit of vanishing lattice spacing the geometry of the classical solutions becomes similar to the geometry of the classical solutions in the dual formulation.

Coming back to our surfaces populated with singular non-Abelian fields they could well be a high-frequency image of classical solution of Yang-Mills theory in the dual representation. This guess cannot be supported however by any straightforward derivation.

\section{Open questions. Conclusions}

As we have demonstrated above, different approaches to magnetic degrees of freedom converge to a common and highly non-trivial picture. In particular, the dual models [14] relate percolation of the magnetic strings to the prediction that they should carry density of the topological charge. The surface operators [2] provide us with an adequate construct to describe such surfaces endowed with both gauge fields and 
their duals. On the lattice, one does observe percolating surfaces, with tension in physical units and endowed with topological charge.

In this note we emphasized that the monopole mechanism of confinement, whatever it means in detail, assumes significant role to be played by singular non-Abelian fields. Indeed, 'electric' degrees of freedom are introduced directly in the Lagrangian, while 'magnetic degrees of freedom' are emerging through violations of Bianchi identities. Hence magnetic degrees of freedom are associated with singular fields.

The construction is well understood and known since long [7] in the Abelian case. One can either start with a gauge field plus a scalar, Higgs field, or one can start with pure gauge field but admit singular field configurations, monopoles. Then these singular fields, or defects can be traded for a scalar field and one comes back to an effective Higgs-like formulation.

In the non-Abelian case monopoles, or $1 \mathrm{~d}$ defects do not match the group structure [9, 18]. Instead, one should look for classification of singular fields consistent with the non-Abelian symmetry. As is argued in fact recently [2] such defects are $2 \mathrm{~d}$ dimensional surfaces with non-Abelian fields living on them. In other words, the surfaces are endowed with densities of action and of topological charge. We argued that such surfaces can be profiled by $1 \mathrm{~d}$ defects, or non-Abelian monopoles, whose field is not spherically symmetric but rather line-like.

For the defects to become dynamical degrees of freedom there should be a fine tuning which allows infrared and ultraviolet scales to coexist. Such fine tuning is well understood in the Abelian case, (see Eq. (6) ). A central point: for the dualsuperconductor mechanism of confinement to be relevant, similar fine tuning should be realized in the non-Abelian case as well. This time, however, it is self-tuning between the action and entropy of the $2 \mathrm{~d}$ surfaces. No explicit form of the fine tuning is known theoretically but coexistence of the two scale is seen in the lattice simulations.

Also, there should be consistency between dynamics in four dimensions, or of the original Yang-Mills fields and dynamics on the defects world-sheet. Let us conclude with an example of such a consistency check. For dynamics of a particle (or $1 \mathrm{~d}$ defect) to be in fact independent on the ultraviolet scale the action associated with the 'monopoles' should be approximately

$$
L \cdot M_{\text {non-Abelian }} \approx L \cdot \frac{\ln 7}{a},
$$

see Eq. (6). And this has been checked on the lattice [25]. On the other hand, the radiative mass (37) is associated now with the non-Abelian fields which live not in the bulk but on the world-sheet (see discussion above). Which seems to be also true on the lattice, for references and review see [13]. The condition (37) replaces the quantization condition for the magnetic field in case of the non-Abelian monopoles living on the surfaces. 


\section{Acknowledgments}

We are thankful to M.N. Chernodub, A.S. Gorsky, F.V. Gubarev, Ph. de Forcrand for useful discussions. This note was worked out mostly during the time when both authors were participants to the workshop "Non-Perturbative Methods in Strongly Coupled Gauge Theories" at the Galileo Galilei Institute (Florence). We are thankful to the GGI for the invitation and hospitality.

\section{References}

[1] Paul Dirac, "Quantised Singularities in the Electromagnetic Field", Proc. Roy. Soc. A 133, (1931) 60.

[2] S. Gukov, E. Witten, "Gauge Theory, Ramification, And The Geometric Langlands Program", arXiv:hep-th/0612073;

E. Witten, Fortsch. Phys., 55 (2007) 545.

[3] R. Savit, Rev. Mod. Phys. 52 (1980) 453.

[4] A.M. Polyakov, "Gauge Fields and Strings", Harvard Academic Publishers, (1987);

J. Ambjorn, "Quantization of geometry", arXiv:hep-th/9411179.

[5] M.N. Chernodub, F.V. Gubarev, M.I. Polikarpov, A. I. Veselov, Progr. Theor. Phys. Suppl., 131. 309 (1998), arXiv:hep-lat/9802036;

A. Di Giacomo, Progr. Theor. Phys. Suppl., 131161 (1998, arXiv:hep-lat/9802008;

T. Suzuki, Progr. Theor. Phys. Suppl., 131633 (1998).

[6] J. Greensite, Prog. Part. Nucl. Phys., 51 (2003) 1, arXiv:hep-lat/0301023.

[7] A.M. Polyakov, Phys. Lett., B59 (1975) 82 ;

T. Banks, R. Myerson, J. B. Kogut, Nucl. Phys., B129 (1977) 493;

H. Shiba, T. Suzuki, Phys. Lett., B333 (1994) 461, arXiv:hep-lat/9404015;

A. Di Giacomo, G. Paffuti, Phys. Rev. D56 (1997) 6816, arXiv:hep-lat/9707003.

[8] M. Stone, P. R. Thomas, Phys. Rev. Lett., 41 (1978) 351;

M.N. Chernodub, V.I. Zakharov, Nucl. Phys., $B 669$ (2003) 233, arXiv:hep-th/0211267.

[9] S. R. Coleman, "The Magnetic Monopole Fifty Years Later", published in Erice Subnuclear 1981:21 (QCD161:I65:1981).

[10] M.N. Chernodub, F.V. Gubarev, M.I. Polikarpov, V. I. Zakharov, Nucl. Phys., B600 (2001) 165, arXiv:hep-th/0010265.

[11] A.M. Polyakov, Phys. Lett. 72B (1978) 477; B. Svetitsky, L.G. Yaffe, Nucl. Phys. B210 (1982) 423. 
[12] R. D. Pisarski, Phys. Rev. D62, 111501 (2000); arXiv:hep-ph/0101168.

[13] V.I. Zakharov, Braz. J. Phys., 37 (2007) 165 arXiv:hep-ph/0612342; AIP Conf. Proc., 756 (2005) 182, arXiv:hep-ph/0501011.

[14] A. Gorsky, V. Zakharov, Phys. Rev. D77 (2008) 045017, arXiv:0707.1284 [hepth];

A.S. Gorsky, V.I. Zakharov, A. R. Zhitnitsky, "On Classification of $Q C D$ defects via holography" arXiv:0902.1842 [hep-ph]].

[15] P. Goddard, J. Nuyts, D. I. Olive, Nucl. Phys. B125 (1977) 1.

[16] T. Sakai, Sh. Sugimoto, Prog. Theor. Phys. 113 (2005) 843, arXiv:hep-th/0412141].

[17] G. 't Hooft, Nucl. Phys., B190 (1981) 455.

[18] E. Lubkin, Ann. Phys., 23 (1963) 233.

[19] G. 't Hooft, Nucl. Phys., B153 (1979) 141.

[20] F.V. Gubarev, A.V. Kovalenko, M.I. Polikarpov, S.N. Syritsyn, V.I. Zakharov, Phys. Lett. B574 (2003)136, arXiv:hep-lat/0212003.

[21] A. V. Kovalenko, S. M. Morozov, M. I. Polikarpov, V. I. Zakharov, Phys. Lett. B 648 (2007) 383 [arXiv:hep-lat/0512036];

R. Hollwieser, M. Faber, J. Greensite, U. M. Heller, S. Olejnik, Phys. Rev. D78 (2008) 054508, arXiv:0805.1846 [hep-lat]].

[22] V.I. Zakharov, "Matter of resolution: From quasiclassics to fine tuning.", in Sense of Beauty in Physics: Miniconference in Honor of Adriano Di Giacomo on his 70th Birthday, Pisa, Italy, Jan 2006. arXiv:hep-ph/0602141.

[23] MILC Collaboration (C. Aubin et al.), Nucl. Phys. Proc. Suppl. 140 (2005) 626, arXiv:hep-lat/0410024;

E.M. Ilgenfritz et al., Phys. Rev. D76 (2007) 034506, arXiv:0705.0018.

[24] A. A. Belavin, V. A. Fateev, A. S. Schwarz and Yu. S. Tyupkin, Phys. Lett. B83 (1979);

V.A. Fateev, I.V. Frolov, A.S. Shvarts, Nucl. Phys. B154 (1979)1.

[25] V.G. Bornyakov et al., Phys. Lett., B537 (2002) 291, arXiv:hep-lat/0103032]. 